\title{
NOISE TOLERANT COLOR IMAGE SEGMENTATION USING SUPPORT VECTOR MACHINE
}

\author{
R. Gnana Vargin", R.V.V. Krishna ${ }^{2}$ \\ ${ }^{1}$ P.G. Student, Electronics \& communication Engineering, Sri Sai Aditya institute of science and technology \\ ${ }^{2}$ Associate professor, Electronics \& communication Engineering, Sri Sai Adithya institute of science and technology
}

\begin{abstract}
Image segmentation is an important tool in image processing and can serve as an efficient front end to sophisticated algorithms. In this paper we present a noise tolerant color image segmentation using pixel wise support vector machine classification(SVM).Firstly the pixel level color feature and texture feature of the image, which is used as input of SVM model (classifier) are extracted through the local homogeneity model and gabor filter. Then the SVM model (classifier) is trained by using PFCM which works efficiently even for noisy images with the extracted pixel level features. Finally the color image is segmented with the trained SVM model (classifier).Experimental evidence shows that the proposed method has a very effective segmentation results and computational behavior. The clustering techniques for color image segmentation using FCM and PFCM are being analyzed. The objective of this paper is to compare the clustering techniques for color images using peak-signal to noise ratio (PSNR), Accuracy, convergence rate, specificity and sensitivity.
\end{abstract}

Keywords: segmentation, FCM, PFCM, SVM classifier, PSNR, Accuracy, convergence rate, specificity and sensitivity. ****

\section{INTRODUCTION}

Image segmentation is an important and challenging problem in image analysis and high level image interpretation such as robot vision, object recognition and medical images[1]. The goal of image segmentation is partition of image in to a set of disjoint regions with uniform and homogenous attributes such as intensity, color, texture etc.., Image segmentation approaches can be divided in to four categories thresholding[2,3], clustering[4-6], edge detection[7-9] and region extraction[10-12]. In this paper, a clustering [13] based noise resistant method for image segmentation [14-15] using support vector machines will be studied.

In this paper we propose a noise resistant color image segmentation using pixel wise support vector machine classification[16-17].Firstly the pixel-level color feature and texture feature of the image with added noise, which is used as input of SVM model (classifier) are extracted through local homogeneity model and Gabor filter. Then the SVM model (classifier) is trained by using PFCM which is more robustness to noise with the extracted pixel-level features. Finally the color image is segmented with the trained SVM model(classifier).This technique not only can take advantage of local information of color image but also the ability of SVM classifier. Simulation results shows that the proposed method achieves good segmentation results by handling any amount of noise by adjusting penalty coefficient. Performance measures like PSNR, Segmentation accuracy; Specificity and Sensitivity have been discussed in this paper.
The rest of the paper is organized as follows section 2 describes the pixel level and color feature extraction from image with added noise, section 3 describes the basic theory behind SVM ,section 4 describes the description of noise resistant color image segmentation. Section 5 describes the simulation results regarding performance measures of this method, section 6 concludes this discussion.

\section{PIXEL LEVEL COLOR AND TEXTURE} FEATURE EXTRACTION

\subsection{Pixel Level Color Feature Extraction [18]}

Here each pixel level of the image with added noise is treated as homogenous region corresponding to an object .The problem of image segmentation is treated as a classification task and the goal of segmentation is to assign a label to individual pixel or a region. It is very important to extract the effective pixel-level image feature .Here we extract the effective pixel-level color and texture feature through local homogeneity and Gabor filter.

Here we use LAB color space to extract pixel level color feature because it is very convenient to measure small color difference.

Let $P_{\mathrm{ij}}=\left(\mathrm{P}_{\mathrm{ij}}{ }^{\mathrm{L}}, \mathrm{P}_{\mathrm{ij}}{ }^{\mathrm{a}}, \mathrm{P}_{\mathrm{ij}}{ }^{\mathrm{b}}\right)$ represent the color components of a pixel at the location (i,j) in an $\mathrm{MXN}$ image and pixel level color feature $\mathrm{CF}_{\mathrm{ij}}{ }^{\mathrm{k}}(\mathrm{k}=\mathrm{L}, \mathrm{a}, \mathrm{b})$ of color component $\mathrm{P}_{\mathrm{ij}}{ }^{\mathrm{k}}(\mathrm{k}=\mathrm{L}, \mathrm{a}, \mathrm{b})$ can be computed as follows:

1. Construct the local image window for computation of pixellevel color feature. 
2. Compute pixel color feature of component $\mathrm{P}_{\mathrm{ij}}{ }^{\mathrm{k}}$ using homogeneity mechanism which is related to local information[19] extracted from image and reflects how uniform a image is.It calculates two components standard deviation and discontinuity of color component $\mathrm{P}_{\mathrm{ij}}{ }^{\mathrm{k}}$.

Standard deviation of color component $\mathrm{P}_{\mathrm{ij}}{ }^{\mathrm{k}}(\mathrm{k}=\mathrm{L}, \mathrm{a}, \mathrm{b})$

$v_{i j}^{k}=\sqrt{\frac{1}{d^{2}}} \sum_{m=i-(d-1 / 2)}^{i+(d-1 / 2)} \sum_{n=j-(d-1 / 2)}^{j+(d-1 / 2)}\left(p_{m n}^{k}-\mu_{i j}^{k}\right)^{2}$

Where $0 \leq i, m \leq M-1,0 \leq j, n \leq N-1$

Where $\mu_{i j}^{k}$ is mean of color component

$p_{i j}^{k}(k=L, a, b) \quad$ with in window $\mathrm{w}_{\mathrm{ij}}$

$\mu_{i j}^{k}=\frac{1}{d^{2}} \sum_{m=i-((d-1 / 2)}^{i+(d+1 / 2)} \sum_{n=j-(d-1 / 2)}^{j+((d-1 / 2)} p_{m n}^{k}$

The discontinuity for color component $\mathrm{P}_{\mathrm{ij}}{ }^{\mathrm{k}}$ is described by edge value, There are many different edge operators: so be 1,1 a place etc..,

Calculate the discontinuity and use the magnitude

$\mathrm{e}_{\mathrm{ij}}{ }^{\mathrm{k}}(\mathrm{k}=\mathrm{L}, \mathrm{a}, \mathrm{b})$ of the gradient at location (i.j) as the measurement

$\mathrm{e}_{\mathrm{ij}}^{\mathrm{k}}=\sqrt{\left(G_{x}^{k}\right) 2+\left(G_{y}^{k}\right)}$

Where $\mathrm{G}_{\mathrm{x}}{ }^{\mathrm{k}}$ and $\mathrm{G}_{\mathrm{y}}{ }^{\mathrm{k}}$ are composed of gradient components $\mathrm{P}_{\mathrm{ij}}{ }^{\mathrm{k}}(\mathrm{k}=\mathrm{L}, \mathrm{a}, \mathrm{b})$ in $\mathrm{X}$ and $\mathrm{Y}$ dimensions.

\subsection{Texture Feature Extraction}

Texture is one common feature used in image segmentation. It is used in conjunction with color information to achieve better segmentation results than possible with color just alone. To obtain the pixel-level texture feature, we apply gabor filter to image and extract the pixel texture features.

Gabor filter[20] is a class of filters in which a filter of arbitrary orientation and scale is synthesized as linear combination of set of basis filters. The edge located at different orientations and scales in a image are detected by splitting the image in to orientation and scale sub bands obtained by the basis filters.

A Two dimensional Gabor filter

$$
\begin{aligned}
\mathrm{g}(\mathrm{x}, \mathrm{y}) & =\mathrm{h}(\mathrm{x}, \mathrm{y}) \exp (2 \Pi \mathrm{jwx}) \\
& =\frac{1}{2 \pi \sigma_{x} \sigma_{y}} \exp \left(\frac{-1}{2}\left(\frac{x^{2}}{\sigma_{x}^{2}}+\frac{y^{2}}{\sigma_{y}^{2}}\right)\right)
\end{aligned}
$$

Let $\mathrm{I}(\mathrm{x}, \mathrm{y})$ is an image with size $\mathrm{w} \mathrm{x}$ h. The gabor filtered output $\mathrm{G}_{\mathrm{mn}}(\mathrm{x}, \mathrm{y})$ of the image $\mathrm{I}(\mathrm{x}, \mathrm{y})$ is defined as its convolution with the gabor filter $\mathrm{g}_{\mathrm{mn}}(\mathrm{x}, \mathrm{y})$

The main steps of pixel level texture feature extraction procedure is

1. Color space transformation from RGB to Ycbcr color space.

2. Applying Gabor filter to Luminance component Y. It is used to identify the regions with dominant orientations and it is maximum of coefficients that determine the orientation at a given pixel location.

3. Pixel texture feature extraction.

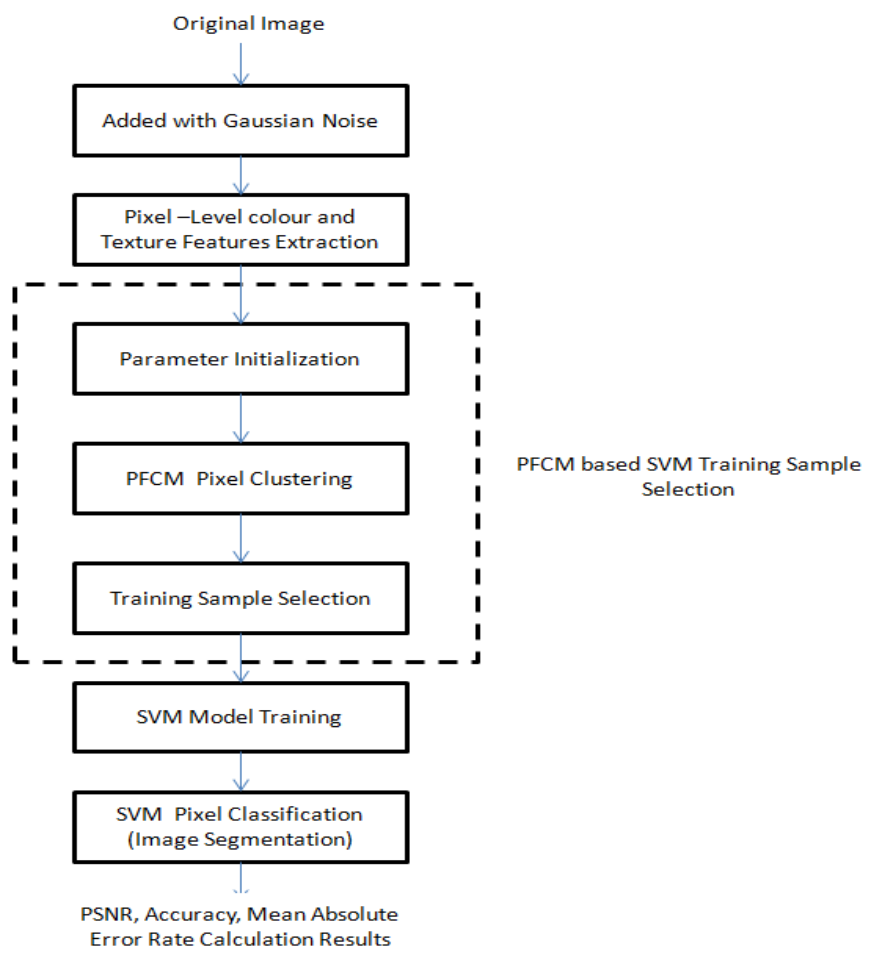

Fig.1. Noise resistant color image segmentation using PFCM based SVM. 


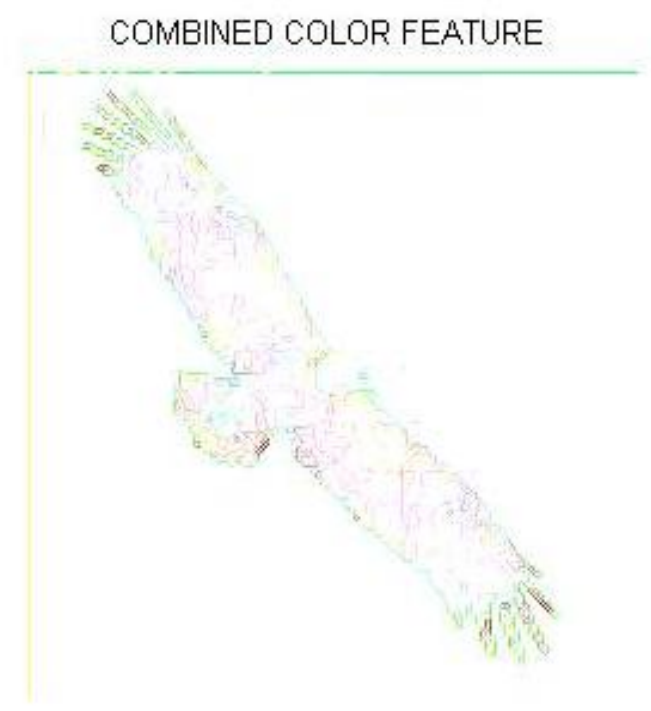

Given a Training data set of 1 points $\left(\mathrm{x}_{\mathrm{i}}, \mathrm{y}_{\mathrm{i}}\right)$ with the input data $\mathrm{x}_{\mathrm{i} \in \mathfrak{K}^{n}}$ and the corresponding target $\mathrm{y}_{\mathrm{i}} \in(-1,+1)$.In feature space SVM model takes the form

$$
\mathrm{Y}(\mathrm{x})=w^{T} \phi(x)+b
$$

Where $\phi(\mathrm{x})$ is a non linear mapping function which maps the input vector in to high dimensional feature space, $\mathrm{b}$ is bias and $\mathrm{w}$ is weighted vector of the same dimensions as feature space.

SVM formulation starts from the assumption that the linear separable case is

$$
\begin{aligned}
& w^{T} x_{i}+b \geq+1\left(y_{i}=+1\right) \\
& w^{T} x_{i}+b \geq-1\left(y_{i}=-1\right)
\end{aligned}
$$

Fig 2 combined color feature extraction

\section{Normalized Gabor Filter output}

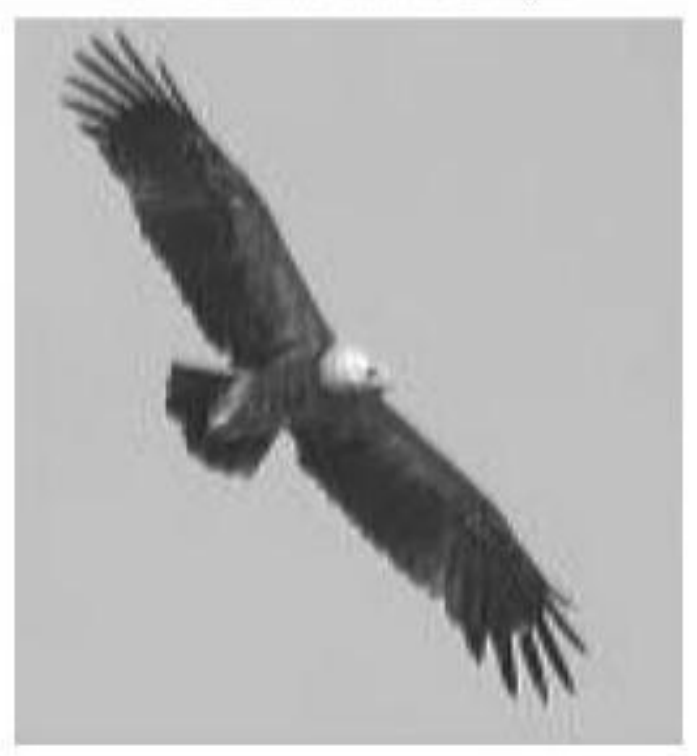

Fig. 3.Texture feature extraction using Gabor filter

\section{THE SUPPORT VECTOR MACHINE (SVM)}

Support vector machines (SVM) $[21,22]$ have been successfully applied in classification and function estimation problems. Standard SVM is used to separate Training data in to two set of classes. The goal of SVM is to find the hyper plane that maximises the minimum distance between any data point as shown in fig. 4

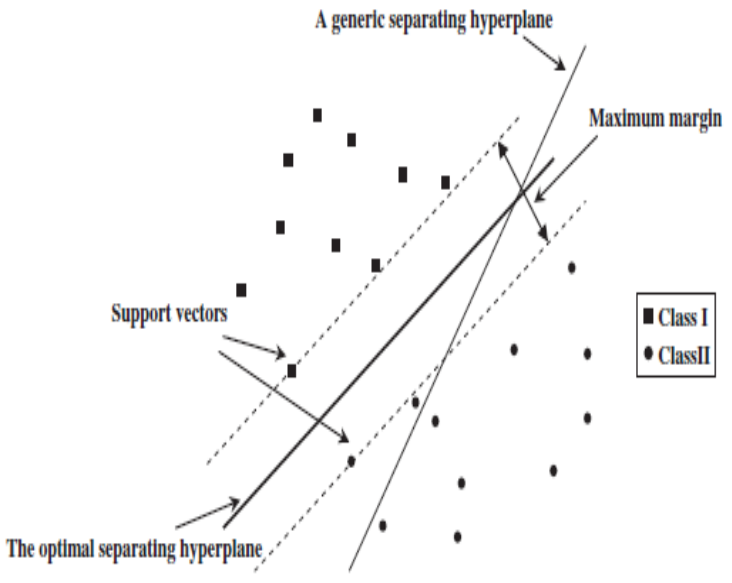

Fig 4 SVM classification (linear)

We need to find among all hyper planes separating the data, an existing maximum $2 / w$ between the classes. In addition to linear classification problems SVM can be applied to non classification problems.

SVM classifiers are used in pattern recognition to partition a feature space derived from the image using data with known labels. A feature space is the range space of any function of the image with the most common feature space being the image intensities them selves.

SVM classifiers are known as supervised methods since they require training data that are manually segmented and then used as reference for automatically segmenting new data. 


\section{SVM Based Classified Image-Penalty FCM}

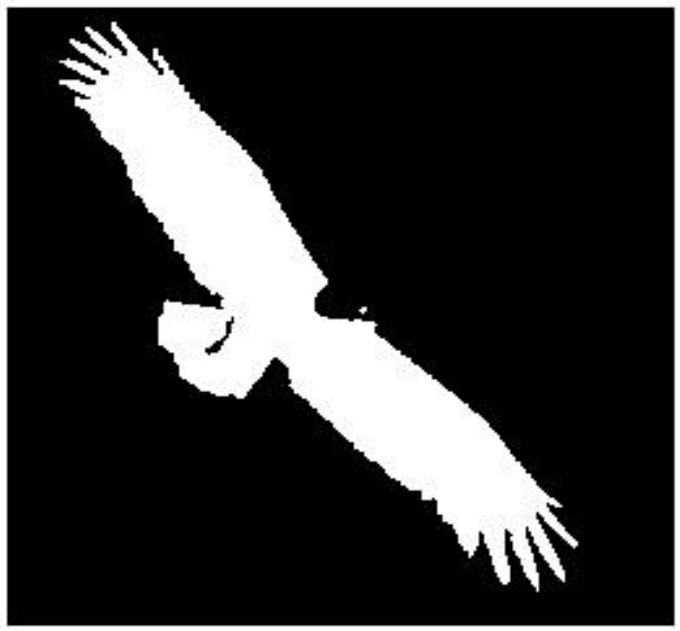

Fig 5 SVM based classified image using penalty FCM.

\section{COLOR IMAGE SEGMENTATION USING}

\section{PFCM}

\subsection{Penalty Fuzzy c-means:}

we propose an enhancement to the FCM algorithm to improve its robustness to noise $[23,24]$.The improvement is formulated by directly placing a new term in to the objective function that constraints the behavior of the membership function such that membership value at each pixel depends not only on the data at the pixel ,but also on the central value of the data set. The additional term determines the amount of smoothness that is positioned up on the member ship function and is determined empirically. The proposed algorithm improves robustness to noise, outliers and retains simplicity. The objective function without penalty term is given as follows:

$$
J_{F C M}=\sum_{K=1}^{n} \sum_{i=1}^{c}\left(\mu_{i k}^{q}\right) d^{2}\left(x_{k}, v_{i}\right)
$$

Where $\mathrm{X}=\left\{x_{1}, x_{2} \cdots \cdots x_{n}\right\}$ set of data

$\mathrm{c}-$ no of clusters $2 \leq \mathrm{c}<\mathrm{n}$

$\mathrm{u}_{\mathrm{ik}}$-degree of membership of $\mathrm{x}_{\mathrm{k}}$ in $\mathrm{i}^{\text {th }}$ cluster.

q-weighing membership on each fuzzy member ship

$\mathrm{v}_{\mathrm{i}}-$ centre of $\mathrm{i}^{\text {th }}$ cluster

$d^{2}\left(x_{k}, v_{i}\right)$ - distance between object $x_{k}$ and cluster center $v_{i}$

The technique presented in this paper is to incorporate neighbour hood information in to FCM algorithm during classification. The Traditional FCM algorithm does not take in to account the spatial information. PFCM contains the spatial information $[25,26]$ and its membership function is given by
$J P F C M=\sum_{K=1 i=1}^{n} \sum_{i=1}^{c}\left(\mu_{i k} q\right) d^{2}\left(x_{k}, v_{i}\right)+\gamma \sum_{k=1}^{n} \sum_{j=1}^{n}\left(\mu_{i k} q\right)\left(1-\mu_{i j}\right)^{q} w_{k j}$

$\mathrm{W}_{\mathrm{kj}}$-spatial structure of given data

$W_{k j}=\left\{\begin{array}{l}1 \text { if } j \text { and } k \text { are neighbours } \\ 0 \text { Otherwise }\end{array}\right.$

\subsection{Implementation:}

The noise resistant color image segmentation using SVM and PFCM can be implemented as:

1. Pixel level color and texture feature extraction

Using local homogeneity model and Gabor filter.

2. PFCM based SVM training sample selection

Set the initial parameters such as no. of clusters.

a) PFCM algorithm is used to cluster image pixels according to color and texture features,spatial context and membership values can be obtained.

b) Classify image pixels by membership values $\mu_{\mathrm{k}}\left(\mathrm{x}_{\mathrm{i}}, \mathrm{y}_{\mathrm{i}}\right)$ suppose $\mu_{\mathrm{j}}\left(\mathrm{x}_{\mathrm{i}}, \mathrm{y}_{\mathrm{i}}\right)=\max \left(\left(\mu_{1}\left(\mathrm{x}_{\mathrm{i}}, \mathrm{y}_{\mathrm{i}}\right), \ldots \ldots \mu_{\mathrm{c}}\left(\mathrm{x}_{\mathrm{i}}, \mathrm{y}_{\mathrm{i}}\right)\right.\right.$ then image pixel at $\left(\mathrm{x}_{\mathrm{i}}, \mathrm{y}_{\mathrm{i}}\right)$ belongs to $\mathrm{j}^{\text {th }}$ cluster

c) For image pixels in $\mathrm{j}^{\text {th }}$ cluster some pixels are chosen as training samples remaining are used as test samples.

3. SVM model training

Train the SVM classifier using training samples created in previous step.

4. SVM pixel classification

Combine test set and training set to get segmentation result.

INPUT IMAGE

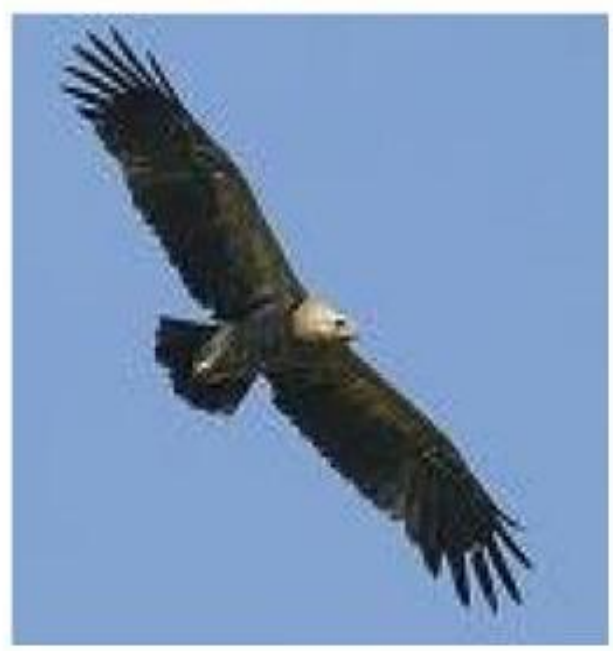

Fig 6 input image 


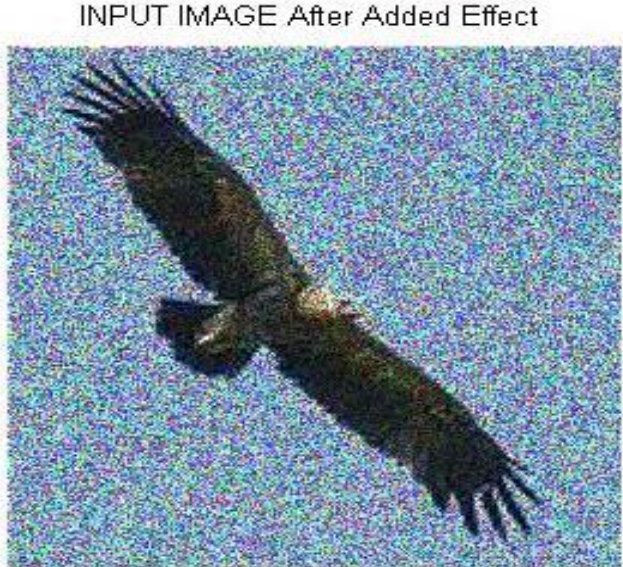

Fig 7 input image after addition of Gaussian noise

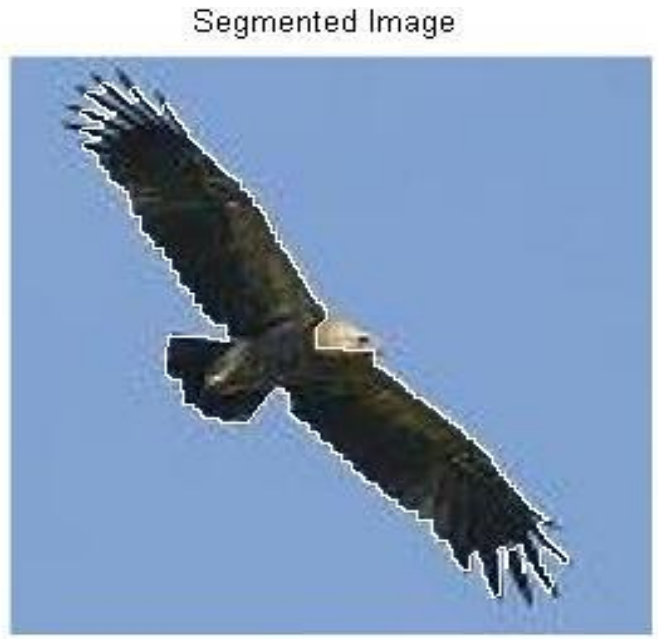

Fig 8 segmented image

\section{PERFORMANCE MEASURES}

AND SIMULATION RESULTS:

1. Peak signal to noise ratio[27,28]: In order to evaluate the performance of different segmentation methods, image quality measurement is required and is known as peak signal to noise ratio.

The PSNR and mean absolute error are two error metrics used to compare quality of image.

$$
\operatorname{PSNR}=20 \log _{10}\left(\frac{255^{2}}{M A E}\right)
$$

2. Sensitivity: It measures the proportion of actual positives which are correctly identified. It relates to the test's ability to identify positive results.
Sensitivity $=\frac{\text { no of true positives }}{\text { no of true positives }+ \text { no of false negatives }}$

3. Specificity: measures the proportion of negatives which are correctly identified..It relates to the ability of the test to identify negative results.

Specificity $=\frac{\text { no of true negatives }}{\text { no of true negatives }+ \text { no of false positives }}$

4. Segmentation Accuracy: segmentation accuracy determines the success or failure rate of computer analysis procedures.

5. Convergence Rate: It is defined as the time required for the execution of the clustering technique.

Simulation Results:

\begin{tabular}{|l|l|l|l|l|}
\hline $\begin{array}{l}\text { Performance } \\
\text { measures }\end{array}$ & K-means & $\begin{array}{l}\text { Rough } \\
\text { K-means }\end{array}$ & FCM & PFCM \\
\hline PSNR & 96.345 & 92.667 & 84.365 & 72.2592 \\
\hline Accuracy & 78.57 & 85.7 & 89.6 & 91.6 \\
\hline $\begin{array}{l}\text { Convergence } \\
\text { rate(sec) }\end{array}$ & 15.82 & 14.402 & 12.063 & 10.583 \\
\hline sensitivity & 83.33 & 86.07 & 91.53 & 95.62 \\
\hline specificity & 86.67 & 89.86 & 92.10 & 96.74 \\
\hline
\end{tabular}

\section{CONCLUSIONS}

Experimental results show that the proposed method is effective and more robust to noise than conventional FCM algorithm. And also the convergence rate, sensitivity, PSNR, specificity and accuracy have been improved. A large value of $\gamma$ is needed to perform segmentation technique on a high amount of noisy images. Future work will focus on adaptively deciding the penalized parameter of this algorithm with out compensating for the texture features, intensity etc..,

\section{REFERENCES}

[1]. Dhawan, A. P., "A Review on Biomedical Image Processing and Future Trends," Computer Methods and Programs in Biomedicine, Vol. 31, No.3-4, 1990, pp.141-183.

[2]. M. Madhubanti, C. Amitava, A hybrid cooperativecomprehensive learning based PSO algorithm for image segmentation using multilevel thresholding, Expert Systems with Applications 34 (2) (2008) 1341-1350.

[3]. M.E. Yuksel, M. Borlu, Accurate segmentation of dermoscopic images by image thresholding based on type-2 fuzzy logic, IEEE Transactions on Fuzzy Systems 17 (4) (2009) 976-982. 
[4]. X.Y. Zeng, Y.W. Chen, Z. Nakao, H.Q. Lu, Texture representation based on pattern map, Signal Processing 84 (3) (2004) 589-599.

[5]. Guan-Yu Chen, Ying-Cheng Chen, et al., Template-based automatic segmen- tation of drosophila mushroom bodies, Journal of Information Science and Engineering 24 (1) (2008) 99-113.

[6]. X. Wang, Y. Sun, A color- and texture-based image segmentation algorithm, Machine Graphics \& Vision 19 (1) (2010) 3-18.

[7]. R. He, S. Datta, B.R. Sajja, Generalized fuzzy clustering for segmentation of multi-spectral magnetic resonance images, Computerized Medical Imaging and Graphics 32 (5) (2008) 353-366.

[8]. J. Shi, J. Malik, Normalized cuts and image segmentation, IEEE Transactions on Pattern Analysis and Machine Intelligence 22 (8) (2000) 888-905.

[9]. P. Bao, L. Zhang, X. Wu, Canny edge detection enhancement by scale multiplication, IEEE Transactions on Pattern Analysis and Machine Intelli- gence 27 (9) (2005) 1485-1490.

[10]. C. Christoudias, B. Georgescu, P. Meer, Synergism in low-level vision, in: Proceedings of the 16th International Conference on Pattern Recognition, vol. IV, Quebec City, Canada, August 2002, pp. 150-155.

[11]. Kuo-Liang Chung, Wei-Jen Yang, Wen-Ming Yan, Efficient edge-preserving algorithm for color contrast enhancement with application to color image segmentation, Journal of Visual Communication and Image Representation 19 (5) (2008) 299-310.

[12]. O.G. Ugarriza, L. Saber, E. Vantaram, et al., Automatic image segmentation by dynamic region growth and multiresolution merging, IEEE Transactions on Image Processing 18 (10) (2009) 2275-2288.

[13]. A Comparative Study of Color Segmentation Using Hard, Fuzzy, Rough Set Based Clustering Techniques. ISSN 2277-3061

[14]. A Survey on Image Segmentation through Clustering Algorithm International Journal of Science and Research (IJSR), India Online ISSN: 2319-7064

[15]. R.C. Gonzalez, R.E. Woods, Digital Image Processing, Prentice-Hall, Englewood Cliffs, NJ,2002.

[16]. Color image segmentation using pixel Wise supportvector machine classification Xiang-Yang Wanga,b,n, TingWanga, JuanBu, IEEE Transactions on pattern recognition.

[17]. S.R. Kannan a,n, R.Devi a, S.Ramathilagam b, K.Takezawa Effective FCM noiseclustering algorithms in medicalimages.

[18]. J.E. Francisco, D.J. Allan, Benchmarking image segmentation algorithms, International Journal of Computer Vision 85 (2) (2009) 167-181.

[19]. R. Unnikrishnan, C.E. Pantofaru, M. Hebert, Toward objective evaluation of image segmentation algorithms, IEEE Transactions on Pattern Analysis and Machine Intelligence 29

(6) (2007) 929-943.
[20]. C. Liu., Gabor-based Kernel PCA with fractional power polynomial models for face recognition, IEEE Transactions on Pattern Analysis and Machine Intelligence 26 (5) (2004) 572581.

[21]. Z. Xue, L. Long, S. Antani, G.R. Thoma, J. Jeronimo, Segmentation of mosaicism in cervicographic images using support vector machines, Proceedings of SPIE Medical Imaging 7259 (1) (2009) 72594X-72594X-10.

[22]. B. Sch“ olkopf, A.J. Smola, in: Learning with Kernels: Support Vector Machines, Regularization, Optimization, and Beyond, MIT Press, Cambridge, MA, 2002.

[23]. Image segmentation by fuzzy c-means clustering with a novel penalty term Yong Yang Computing and Informatics, Vol. 26, 2007, 17-31.

[24]. Jiashun Chen,An Insensitivity Fuzzy C-means Clustering Algorithm Based on Penalty Factor.

[25]. Y.A. Tolias and S.M. Panas, On applying spatial constraints in fuzzy image clustering using a fuzzy rule based system., IEEE Signal Processing Letters 5, 245.247(1998).

[26]. Y.A. Tolias and S.M. Panas, Image segmentation by a fuzzy clustering algorithm using adaptive spatially constrained membership functions., IEEE Trans. Systems, Man, Cybernet. A28, 359.369 (1998).

[27]. Comparison and Analysis of fuzzy Clustering techniques in terms of accuracy and PSNR. International Journal of Advanced research in computer science.

[28]. F.Meyer, "Color Image Segmentation", In IEEE International Conference on Image Processing and its Applications, 1995. 